\title{
Special issue: Selected papers from the 26th International Conference on Concurrency Theory (CONCUR 2015)
}

\author{
Luca Aceto $^{1}$. David de Frutos Escrig ${ }^{2}$
}

Published online: 19 January 2017

(C) Springer-Verlag Berlin Heidelberg 2017

The February, April and June 2017 issues of Acta Informatica are devoted to presenting carefully refereed, full versions of selected papers from CONCUR 2015, the 26th International Conference on Concurrency Theory, whose program committee we had the pleasure of co-chairing.

The conference was held in the period 1-4 September 2015 at the Facultad de Matemáticas, Universidad Complutense de Madrid, and was co-located with the 12th International Conference on Quantitative Evaluation of SysTems (QEST 2015), the 13th International Conference on Formal Modeling and Analysis of Timed Systems (FORMATS 2015), the 10th International Symposium on Trustworthy Global Computing (TGC 2015), the International Symposium on Web Services, Formal Methods and Behavioural Types (WS-FM/BEAT 2015), and seven workshops.

The eight papers that appear in this issue and the two following ones were selected based on the referee reports we received on their conference versions and on the ensuing discussion among the program committee members. They witness the high quality of the scientific program of CONCUR 2015 and reflect current trends in research in concurrency theory.

The February 2017 issue of Acta Informatica, which you are currently reading, contains three papers dealing with games and the role they play in the synthesis of computing systems from their specifications. The contribution by Hunter, Pérez and Raskin deals with the syn-

Both authors were partly supported by the Project 001-ABEL-CM-2013 within the NILS Science and Sustainability Programme. Luca Aceto also acknowledges the partial support of the projects 'Nominal Structural Operational Semantics' (Grant No.: 141558-051) and 'TheoFoMon: Theoretical Foundations for Monitorability' (Grant No.: 163406-051) of the Icelandic Research Fund.

Luca Aceto

luca@ru.is

David de Frutos Escrig

defrutos@sip.ucm.es

1 School of Computer Science, ICE-TCS, Reykjavik University, Reykjavík, Iceland

2 Department of Informatic Systems and Computation, Universidad Complutense de Madrid, Madrid, Spain 
thesis of strategies in two-player zero-sum games of infinite duration that minimize the regret of one of the players - that is, strategies that minimize the difference between that player's actual payoff and the payoff she could have achieved if she had known the strategy of her opponent in advance. The paper by Brenguier, Raskin and Sankur introduces a novel rule for synthesis of reactive systems, which is applicable to systems consisting of any number of components, each having its own goal. Finally, the article by Brihaye, Geeraerts, Haddad and Monmege gives the first pseudo-polynomial-time algorithm for total-payoff games in the presence of arbitrary weights.

The April 2017 issue of Acta Informatica will consist of three contributions, from three different areas of research within concurrency theory. The paper by Bonchi, Petrisan, Pous and Rot proves the soundness of bisimulation up-to techniques within a categorical setting, which allows them to obtain, in a principled way, up-to techniques for a large class of conductively defined predicates modelled as coalgebras. The study by Esparza, Ganty, Leroux and Majumdar establishes the decidability of two classic algorithmic problems in the field of population protocols, by reduction to the reachability problem for Petri nets. The third article in that issue, which is co-authored by Soudjani, Abate and Majumdar, investigates the problem of finite-horizon probabilistic invariance for discrete-time Markov processes over general state spaces. The authors show how to compute a discrete-time, finite-state Markov chain that is an abstraction of a given Markov process, and how to represent such abstractions compactly using dynamic Bayesian networks.

The third and last issue of Acta Informatica devoted to CONCUR 2015 will be published in June 2017 and will report on recent work that contributes to the active research area of session types. It will feature a contribution by Carbone, Montesi, Schürmann and Yoshida proposing a Curry-Howard correspondence between a language for programming multiparty sessions and a generalisation of Classical Linear Logic. The second paper, co-authored by Kouzapas, Perez and Yoshida, presents a notion of characteristic bisimilarity that characterises contextual equivalence in the setting of a higher-order $\pi$-calculus with session types.

We trust that the papers in these issues will give their readers a glimpse of the latest research in concurrency theory.

We take this opportunity to thank the authors who submitted their work to CONCUR 2015, the members of our program committee for the conference and their reviewers, and the colleagues who carefully refereed the papers that appear in this special issue. It has been a humbling experience to work with all of these scientists and we have learned much from this experience.

Last, but not least, we thank Christel Baier, the editor in chief of Acta Informatica, for giving us the opportunity to edit this special issue and Anitha Kamaraj, the Journals Editorial Office Assistant, for her timely and very professional support. 